\title{
Development of CDMA-QAM Rail Transmission Device for Railway Signaling*
}

\author{
Hiroshi MOCHIZUKI**, Sei TAKAHASHI**, Hideo NAKAMURA**, \\ Satoshi NISHIDA***, Minoru SANO ${ }^{* * *}$ and Ryo ISHIKAWA ${ }^{* * *}$ \\ ** Department of Electronics and Computer Science, College of Science and Technology, Nihon University \\ 7-24-1 Narashinodai, Funabashi-shi, Chiba, 274-8501, Japan \\ E-mail: mochizuki@ecs.cst.nihon-u.ac.jp \\ *** Kyosan Electric Mfg. Co., Ltd. \\ 2-29-1 Heian-cho, Yokohama-shi, Kanagawa, 230-0031, Japan
}

\begin{abstract}
To realize high-speed data transmission for railway signaling systems, we propose a novel modulation method combining code-division multiple access (CDMA) and quadrature amplitude modulation (QAM), called the CDMA-QAM method. We evaluated the effectiveness of the proposed method and developed a CDMA-QAM rail transmission system based on DSPs and an FPGA. In addition, we conducted a field trial using an actual rail.
\end{abstract}

Key words : Railway Signaling, Track Circuit, CDMA, QAM, DSP, FPGA

\section{INTRODUCTION}

Railway signaling systems that transmit information via rails have been deployed in many applications, for example, digital automatic train control (ATC) systems for controlling train speed. Since the performance of digital ATC systems depends on the transmission speed, recently there has been a great deal of research aimed at realizing high-speed data transmission (1). Using the rail as a transmission medium has several benefits, such as superior security and cost performance compared with wireless communication methods. However, it is difficult to increase the transmission speed because rails exhibit strong attenuation at frequencies above about $10 \mathrm{kHz}$. Therefore, the present transmission speed of digital ATC using rails is limited to about 300 bps.

To overcome this restriction, we aimed to increase the transmission speed by improving the modulation method. In this paper, we propose a new modulation method combining codedivision multiple access (CDMA) and quadrature amplitude modulation (QAM), named the CDMA-QAM method ${ }^{(2)}$. We evaluated the effectiveness of the proposed method by carrying out computer simulations.

Here we show the hardware architecture of the CDMA-QAM transceiver. We developed a CDMA-QAM receiver based on DSPs and an FPGA, and we conducted an experiment to verify basic functions, such as synchronous detection.

In addition we conducted a field trial using an actual rail. We show spectrum distribution and constellation characteristics obtained from the field trial.

\section{OVERVIEW OF CDMA-QAM METHOD}

First, we explain about principles of CDMA and QAM. CDMA can be realized using spread spectrum (SS) modulation. Figure 1 shows a block diagram of SS modulation. In this figure, the transmitted data is modulated using a spread code in addition to a carrier modulation such as phase shift keying (PSK). As a result of this operation, a spectrum of modulation signal is spread. At the reciever, the transmitted data is recieved by demodulating after a spread code and a carrier were synchronized. Here we assume a transmission on some 


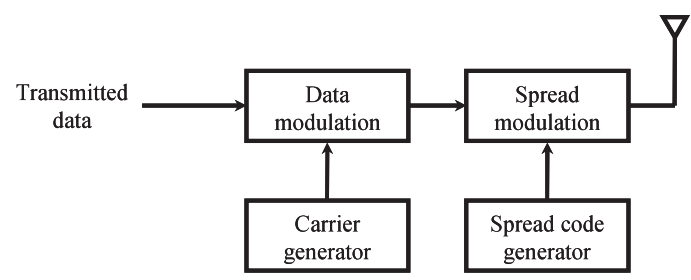

Transmitter

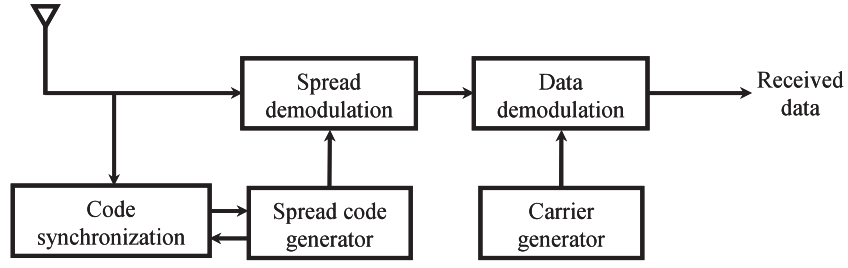

Receiver

Fig. 1 Block diagram of SS modulation.

channels divided by different spread codes. If the cross-correlation between spread codes is small, it is possible to overlap both channels at the same frequency band. It is called CDMA. As a spread code in our proposed method, we applied Walsh code given by the following equation:

$$
\begin{aligned}
& H_{1}=[1] \\
& H_{N}=\left[\begin{array}{ll}
H_{N-1} & H_{N-1} \\
H_{N-1} & -H_{N-1}
\end{array}\right]
\end{aligned}
$$

Each column of the matrix generated by Eq. (1) is given to each channel as spread code. Walsh code has a characteristics that the cross-correlation is zero when all codes are synchronizing. Therefore it is possible to overlap all channels at the same frequency band. This time we applied the transmission on 63 channels given by Walsh codes that has code length of 64 .

On the other hand, QAM is a transmission method that can be realized using two orthogonal carrier signals. One is given by $\cos 2 \pi f_{c} t$, and the other is given by $\sin 2 \pi f_{c} t$. The two carrier signals remain orthogonal in the area of a period

$$
\int_{0}^{T_{c}} \cos 2 \pi f_{c} t \times \sin 2 \pi f_{c} t d t=0
$$

where $T_{c}$ is the period of the carrier signals, and it is equal to inverse of $f_{c}$. By using $\cos 2 \pi f_{c} t$ and $\sin 2 \pi f_{c} t$, QAM signal is given by the following equation:

$$
s(t)=d_{I}(t) \cos \left(2 \pi f_{c} t\right)+d_{Q}(t) \sin \left(2 \pi f_{c} t\right)
$$

A channel in which $\cos 2 \pi f_{c} t$ is used as a carrier signal is generally called an in-phase channel, or I channel, and a channel in which $\sin 2 \pi f_{c} t$ is used as a carrier signal is generally called a quadrature-phase channel, or Q channel. Therefore, $d_{I}(t)$ and $d_{Q}(t)$ are the data in I channel and Q channel, respectively. By the bit number given to amplitudes of $d_{I}(t)$ and $d_{Q}(t)$, there are 16QAM, 64QAM, 256QAM and so on. Figure 2 shows a block diagram of 16QAM. In this figure, 16QAM transmits four bits simultaneously by giving each two bits to I channel and $\mathrm{Q}$ channel ${ }^{(3)}$.

Based on these principles, Fig. 3 shows a block diagram of our proposed method, named CDMA-QAM method. In this method, a QAM symbol allocated based on the multiplexed CDMA signal is transmitted. Specifically, the transmitter first divides the transmitted data into parallel data on 63 channels. 


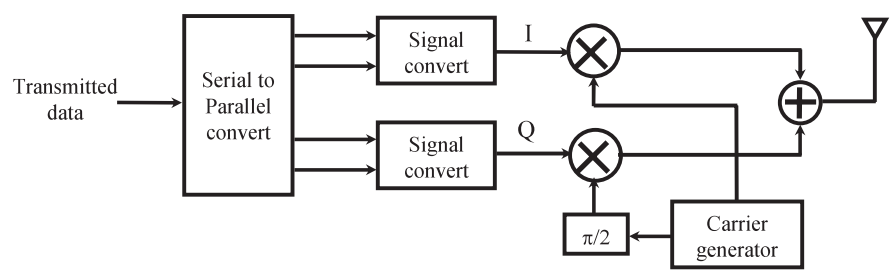

Transmitter

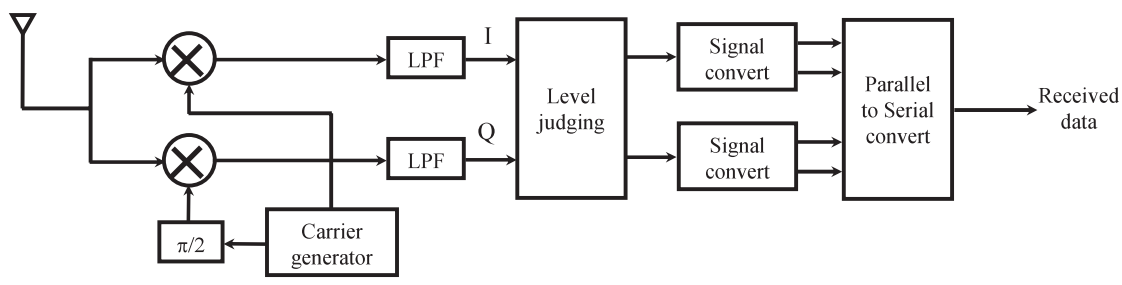

Receiver

Fig. 2 Block diagram of 16QAM.

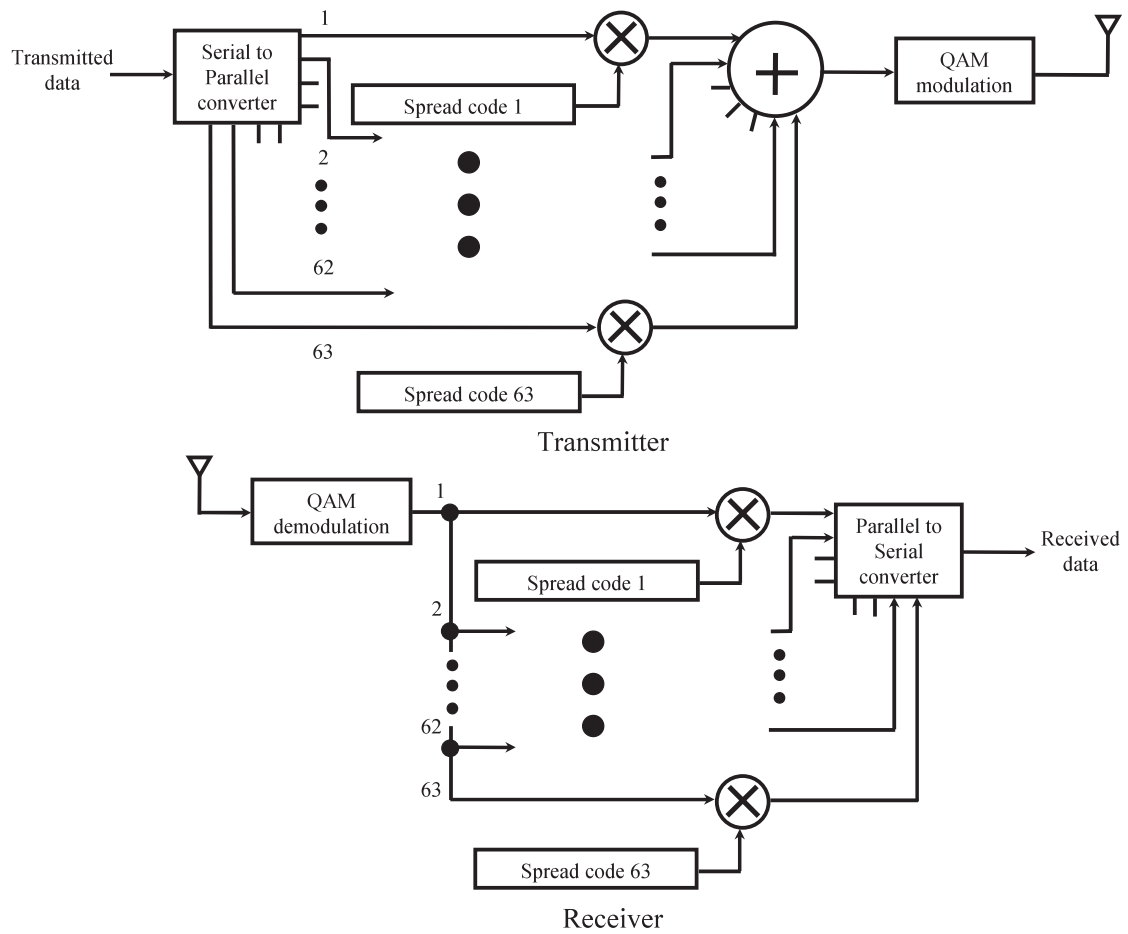

Fig. 3 Block diagram of CDMA-QAM method. 


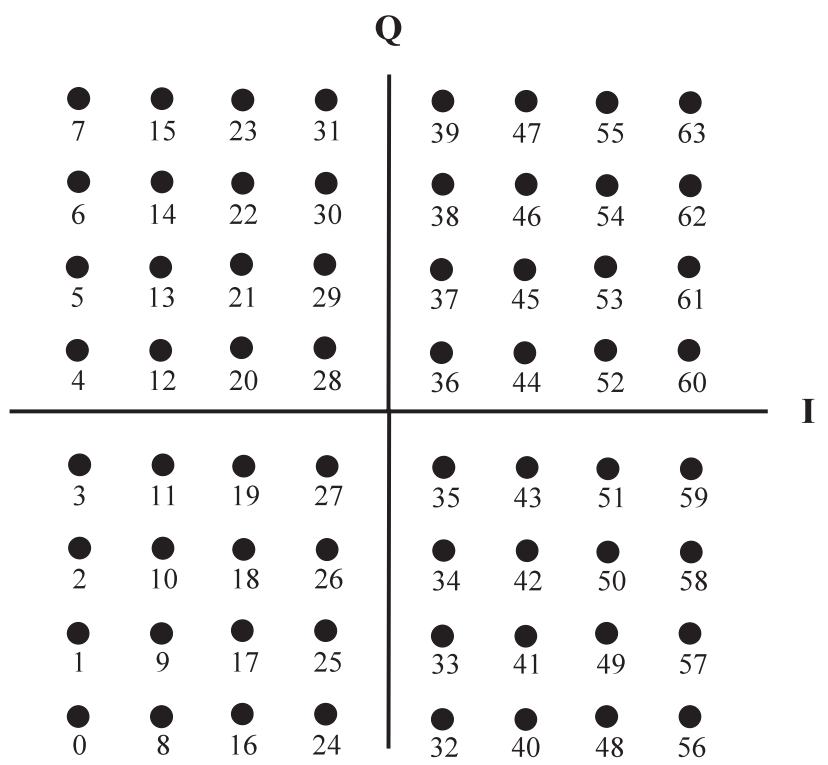

Fig. 4 Amplitude data allocation of each symbol in CDMA-QAM method.

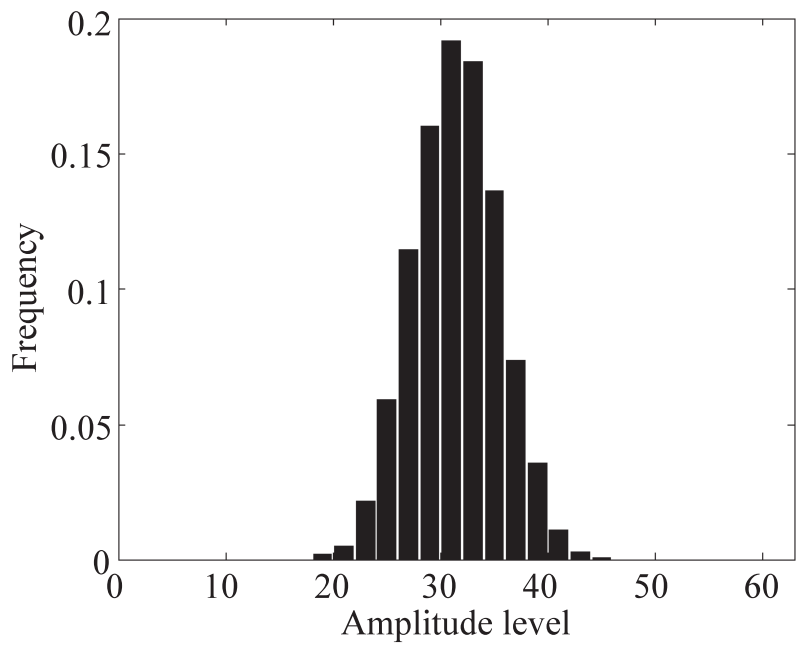

Fig. 5 Frequency distribution of amplitude of multiplexed CDMA signal.

A multiplexed CDMA signal is generated by subjecting all channels to CDMA modulation and adding them. Finally, the multiplexed signal is allocated to 64QAM symbols according to the amplitude of the multiplexed signal, between 0 and 63 , and is transmitted on the transmission line. Here, after the amplitude of the multiplexed signal is converted into binary, the three higher-order bits are allocated to the I channel, and the three lower-order bits are allocated to the $\mathrm{Q}$ channel. In addition, the transmission line shown in Fig. 3 is a track circuit (rail) in this study. We describe about characteristics of a track circuit in the next chapter.

Figure 4 shows amplitude data allocation of each symbol in the CDMA-QAM method. After the receiver demodulates the 64QAM and CDMA signals, it converts the parallel data into received data. A benefit of this proposed method is that CDMA can reduce errors even if they are present with the QAM demodulation. Since QAM does not allocate the transmitted data directly but the multiplexed CDMA signal, so long as the QAM errors can be absorbed by the CDMA demodulation, they can be recovered.

Next, we describe a proposed symbol placement scheme in the CDMA-QAM method. Figure 5 shows the results of analyzing the frequency distribution of the amplitude of the 


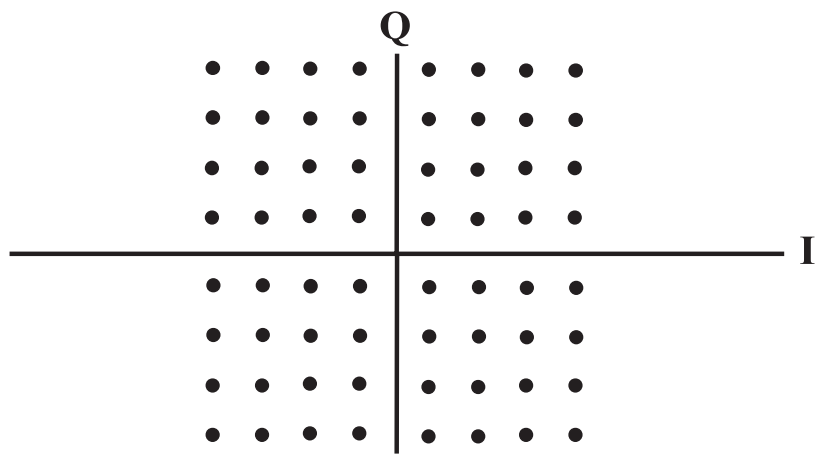

$\mathrm{I}: \mathrm{Q}=1: 1$

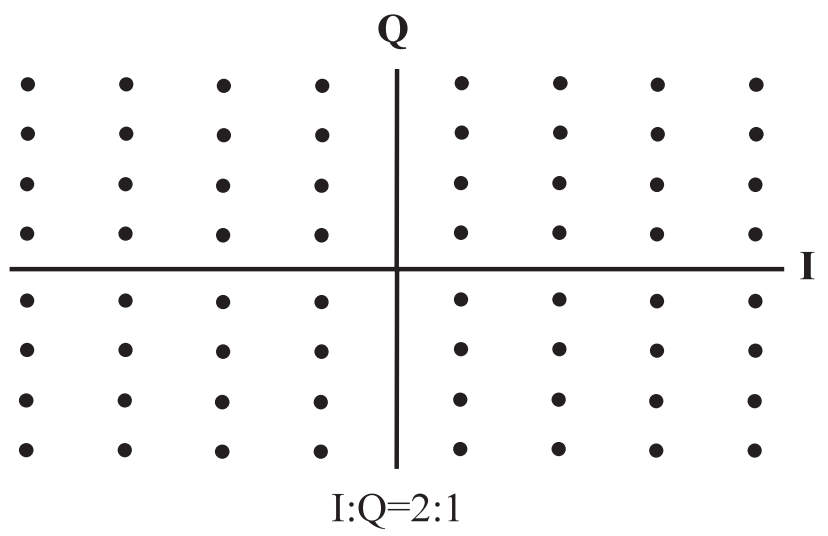

Fig. 6 Symbol placement in CDMA-QAM method.

multiplexed CDMA signal in the transmitter. This figure shows that the frequency distribution is inhomogeneous and centered at 32 (almost the median amplitude value of the multiplexed signal). This means that QAM symbols are centered near the Q-axis. On the other hand, the I channel is more important than the $\mathrm{Q}$ channel for bit error rate (BER) characteristics. This is because the I channel is allocated the three higher-order bits of the multiplexed CDMA signal. Based on this requirement, we designed a symbol placement scheme in which the symbol interval of the I channel is wider than that of the Q channel, as shown in Fig. 6. By using Eq. (3), the transmitted signal is given by the following equation:

$$
s(t)=w_{I} \times d_{I}(t) \cos \left(2 \pi f_{c} t\right)+w_{Q} \times d_{Q}(t) \sin \left(2 \pi f_{c} t\right)
$$

Here $w_{I}$ is the weight of I channel, and $w_{Q}$ is the weight of $\mathrm{Q}$ channel. According to this design, the CDMA-QAM method can improve the BER characteristics without substantially increasing the total electrical power of the system.

\section{APPLICATION TO RAILWAY SIGNALING AND EVALUATION USING COMPUTER SIMULATION}

We applied the proposed CDMA-QAM method to railway signaling, called the CDMAQAM rail transmission system. Table 1 shows the main specifications of the system. Since the Walsh code, which is used as a spread code, includes a code in which all bits are one, we designed this transmission system using 63 channels for all codes except this one.

Based on these specifications, we configured the transmission model using MATLAB which is one of technical programming languages, and carried out computer simulation when the $\mathrm{I} / \mathrm{Q}$ symbol interval rate was varied from $\mathrm{I}: \mathrm{Q}=1: 1$ to $\mathrm{I}: \mathrm{Q}=4: 1$.

Here we describe about track circuit which is used as a transmission line. We can consider a track circuit as a distributed constant circuit shown in Fig. 7. In this figure, $R, L, G$ and $C$ are as follows: 
Table 1 Specifications of CDMA-QAM rail transmission system.

\begin{tabular}{|c|c|}
\hline Parameter & Values \\
\hline Carrier frequency & $4,096 \mathrm{~Hz}$ \\
\hline Spread code & Walsh code \\
\hline The number of multiple access & 63 \\
\hline Transmission speed for each channel & $32 \mathrm{bps}$ \\
\hline Total transmission speed & $2,016 \mathrm{bps}$ \\
\hline Rail length & $1 \mathrm{~km}$ \\
\hline Rail noise & Measured data \\
\hline
\end{tabular}

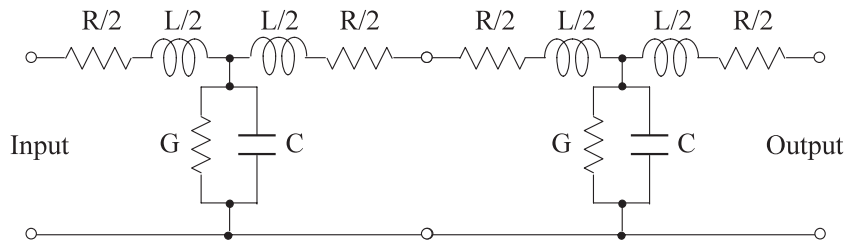

Fig. 7 Equivalent circuit of track circuit.

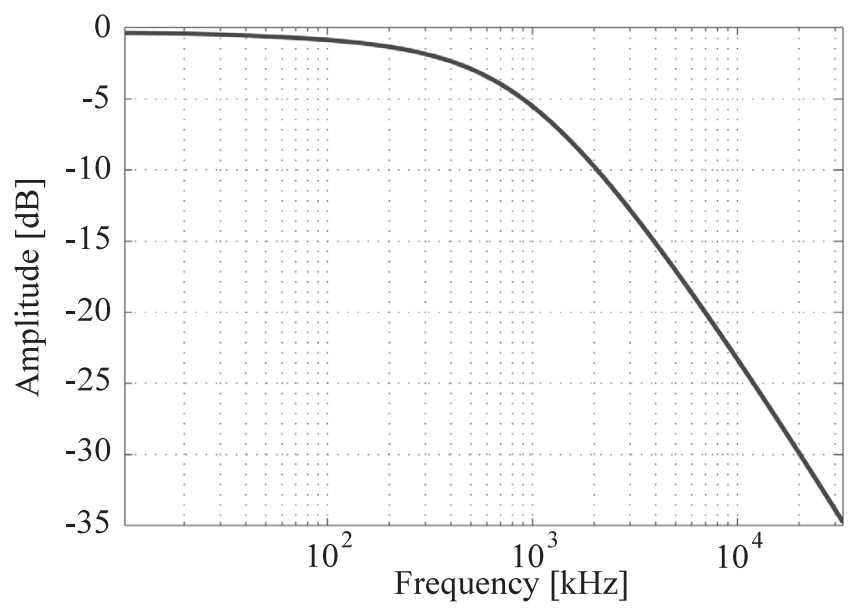

Fig. 8 Frequency response of track circuit.

$R$ : Rail resistance $[\Omega / \mathrm{km}]$

$L$ : Rail inductance $[\mathrm{mH} / \mathrm{km}]$

$G$ : Leakage conductance $[\mathrm{S} / \mathrm{km}]$

$C:$ Capacitance $[\mu \mathrm{F} / \mathrm{km}]$

They are called a track circuit constant. And since a track circuit has a frequency response similar to a low pass filter (LPF) shown in Fig. 8, an audio frequency band is used as a carrier frequency. In this computer simulation, we realized the frequency response of track circuit shown in Fig. 8 by digital filter, and applied to the transmission that we configured.

Figure 9 shows a comparison of constellation characteristics for the different I/Q symbol interval rates. Here the characteristics for $\mathrm{I}: \mathrm{Q}=4: 1$ are normalized according to the scale of the $\mathrm{I}: \mathrm{Q}=1: 1$ characteristics. From this figure, for $\mathrm{I}: \mathrm{Q}=4: 1$, it is possible to reduce the error vector magnitude (EVM) of the I channel, though the EVM of the Q channel is worse than that of $\mathrm{I}: \mathrm{Q}=1: 1$. As mentioned above, since the $\mathrm{I}$ channel is more important than the $\mathrm{Q}$ channel for the BER characteristics, $\mathrm{I}: \mathrm{Q}=4: 1$ has an advantage.

In addition, Fig. 10 shows the BER characteristics of the CDMA-QAM rail transmission system. From this figure, we verified that the BER characteristics improved in proportion to the $\mathrm{I} / \mathrm{Q}$ symbol interval rate. We also showed that $\mathrm{I}: \mathrm{Q}=4: 1$ could realize a BER of $10^{-5}$, which is the standard value for railway signaling, even though the transmission speed was about $2,000 \mathrm{bps}$, which is much higher than currently used transmission speeds. 


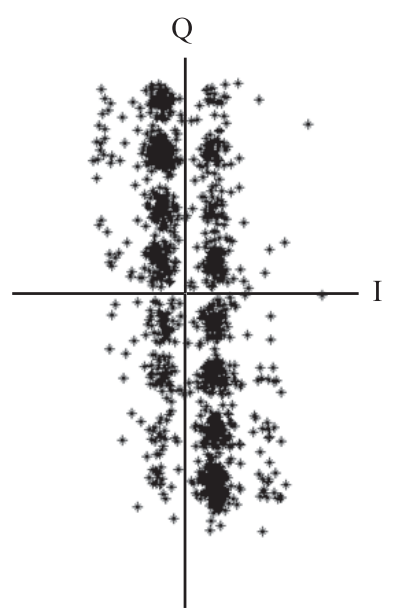

$\mathrm{I}: \mathrm{Q}=1: 1$

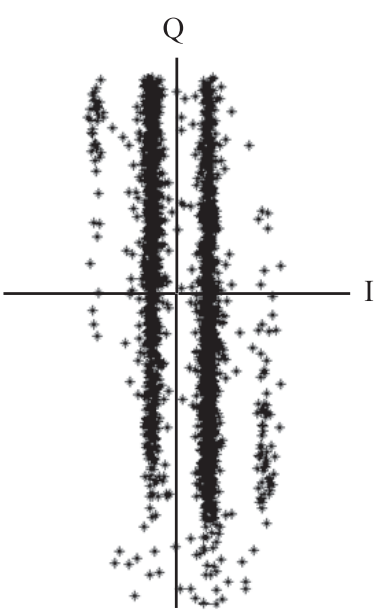

$\mathrm{I}: \mathrm{Q}=4: 1$

Fig. 9 Comparison of constellation characteristics for different I/Q symbol interval rates.

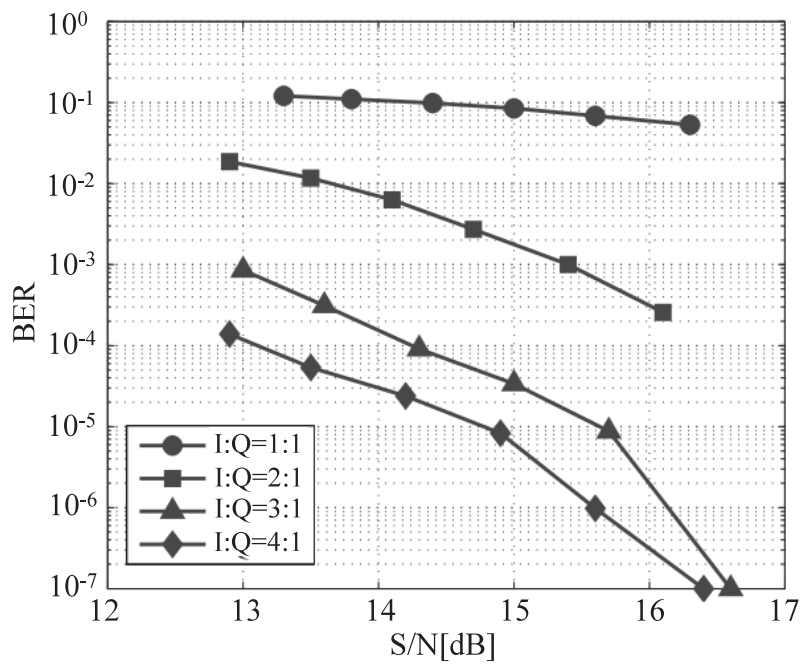

Fig. 10 BER characteristics of CDMA-QAM rail transmission system.

\section{HARDWARE DEVELOPMENT OF CDMA-QAM TRANCEIVER}

\subsection{Hardware architecture}

Figure 11 shows the hardware architecture of the CDMA-QAM transceiver. This figure shows independently developed CDMA and QAM parts. When we compared the transmitter and receiver, the amount of processing in the receiver was much larger than that in the transmitter because the receiver must perform filtering, synchronous detection, and so on. Therefore, we independently developed the CDMA part and the QAM part in the receiver.

Since this transceiver uses the audio-frequency band, we employed DSPs in the transmitter and QAM part of the receiver. On the other hand, we attempted a logic-based design of a matched filter, which is a key device of CDMA, and employed an FPGA.

\subsection{QAM transceiver development using DSPs}

In view of cost, versatility, and so on, we developed the transceiver using a DSP starter kit (DSK). Table 2 shows the specifications of the CDMA-QAM transceiver using DSPs. As mentioned above, since this transceiver uses the audio-frequency band, the standard audio interface of the DSPs was used for connection to the rail. In addition, we used general-purpose I/O (GPIO) for the digital interface between the DSP and the CDMA part of the receiver. 


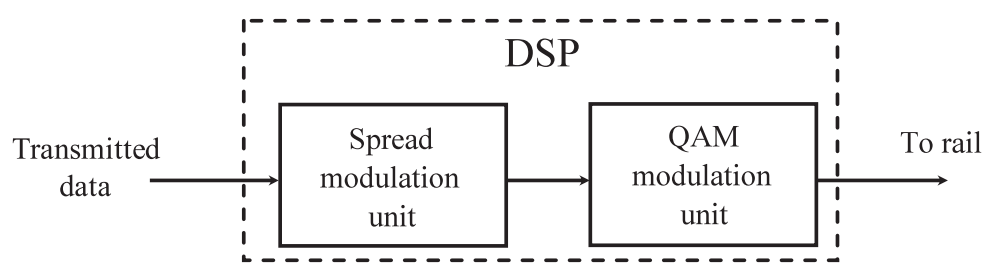

Transmitter

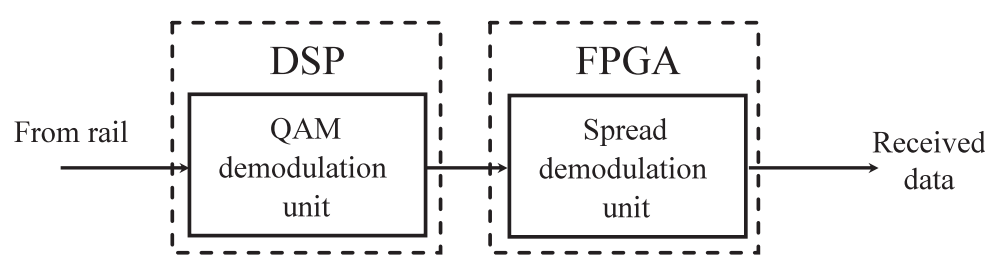

Receiver

Fig. 11 Hardware architecture of CDMA-QAM transceiver.

Table 2 Specifications of CDMA-QAM transceiver.

\begin{tabular}{|c|c|}
\hline Parameter & Values \\
\hline Carrier frequency & $3,000 \mathrm{~Hz}$ \\
\hline The number of multiple access & 63 \\
\hline Transmission speed for each channel & $23.4375 \mathrm{bps}$ \\
\hline Total transmission speed & $1476.5625 \mathrm{bps}$ \\
\hline Sampling frequency & $48 \mathrm{kHz}$ \\
\hline
\end{tabular}

Figure 12 shows the developed transmission device using DSP. We conducted an experiment to verify the basic functions of the CDMA-QAM transceiver with the specifications in Table 2.

As a result, we obtained the CDMA-QAM modulation signal shown in Fig. 13, the GPIO digital output shown in Fig. 14, and the QAM constellation characteristics shown in Fig. 15. As shown in these figures, we verified that this transceiver successfully performed basic functions in real time, such as synchronous detection.

\subsection{CDMA receiver development using an FPGA}

Figure 16 is a matched filter, which is a key device of a CDMA receiver. The structure is the same as a finite impulse response (FIR) filter. It outputs a correlation between a received signal and a spread code serving as a filter coefficient. In addition, when synchronization is assured, transmission data is obtained by picking up the sign bit of the matched filter output.

As mention above, since the number of channels is 64, ideally the CDMA receiver should be capable of demodulating 64 channels all together. Therefore, we attempted to implement this using an FPGA based on optimization of the matched filter shown in Fig. 16.

In Fig. 16, the matched filter includes 64 multipliers. This means that the total number of multipliers is 4,096 for 64 channels. It is impossible to implement this level of processing in FPGA. Therefore, we examined a design that could be implemented without multipliers. Here a spread code that is used as a filter coefficient is 1 or -1 . We can replace multipliers by adders (when the spread code is 1), and subtractors (when the spread code is -1). In addition, since a subtractor is realized by twos complement and an adder, we can replace subtractors with adders.

However, even if the multipliers were replaced by adders, there would be 4,096 adders in the CDMA receiver. Hence, we aimed to further reduce the number of adders. Here the target transmission speed of 2,000 bps is not so fast compared with the $50 \mathrm{MHz}$ clock frequency of the FPGA. Therefore, we employed time-sharing processing. We designed a CDMA receiver 


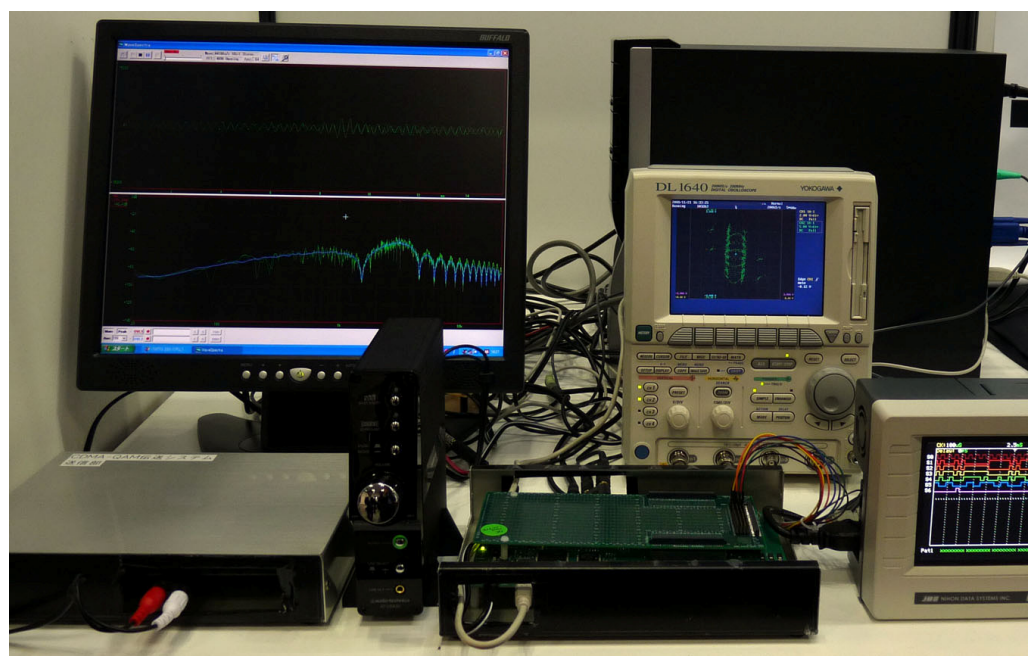

Fig. 12 Developed transmission device using DSP.

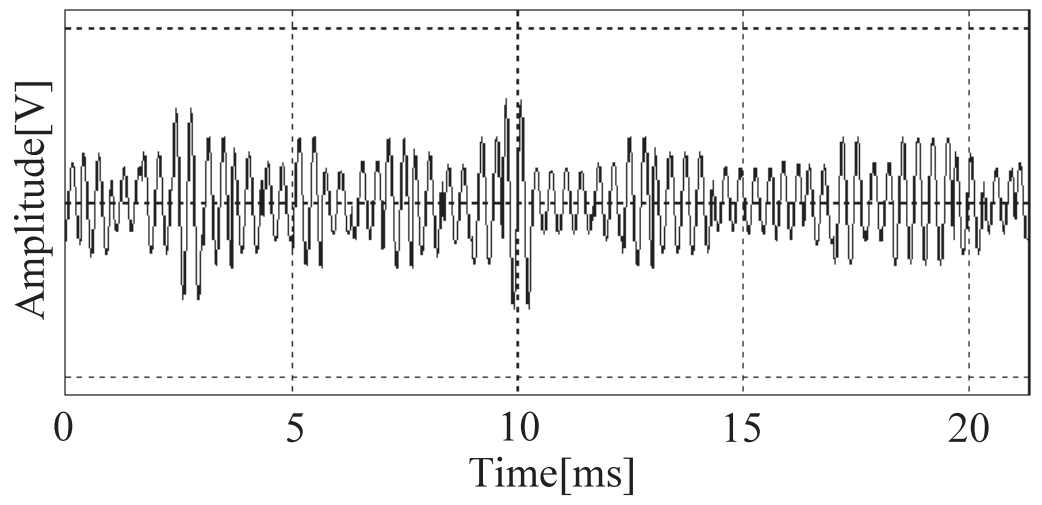

Fig. 13 Waveform of modulation signal.

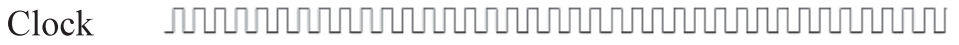

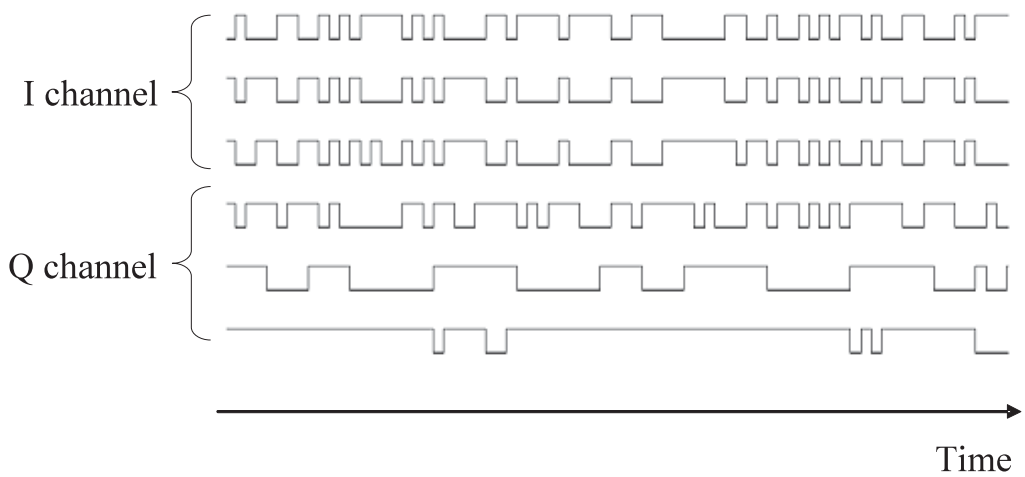

Fig. 14 GPIO digital output in QAM receiver. 


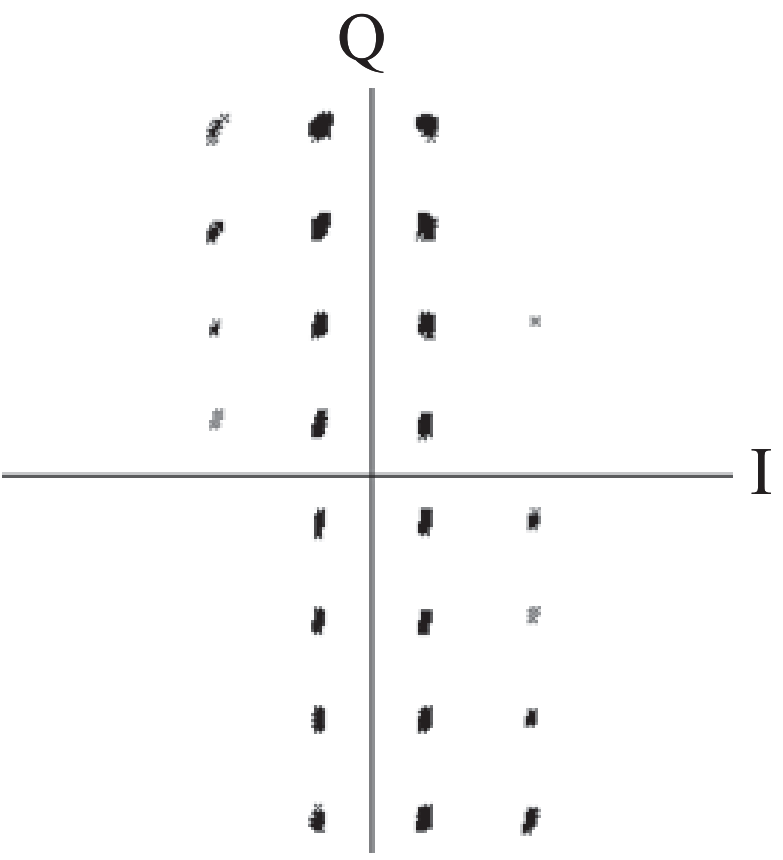

Fig. 15 QAM constellation characteristics.

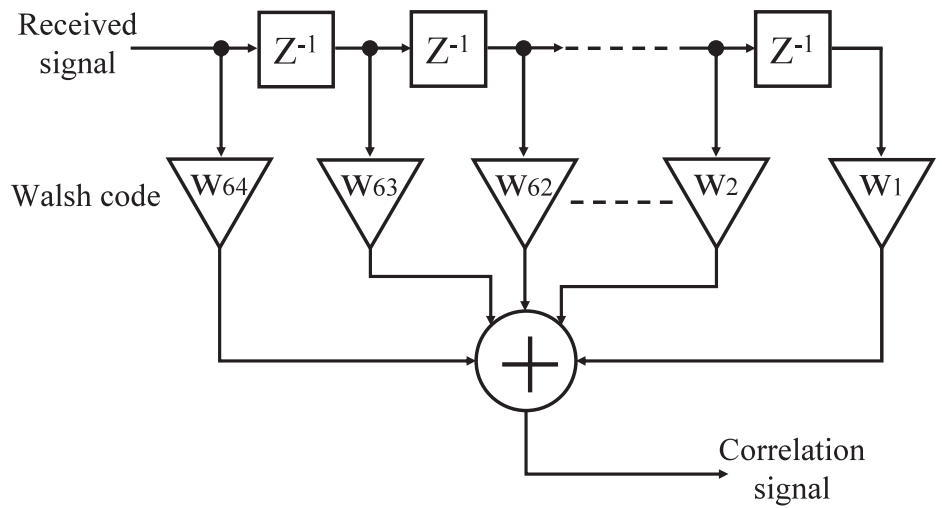

Fig. 16 Block diagram of matched filter.

that includes only one adder and a first-in first-out (FIFO) buffer that has a similar function to the delay devices shown in Fig. 16. In addition, extension to 64 channel processing can be realized by changing the length of the FIFO. As a result of such reconfiguration, we could dramatically reduce the number of hardware devices.

We implemented the CDMA receiver using FPGA shown in Fig. 17. First, we evaluated number of logic elements (LEs). There were 342 LEs, which is much fewer than the 18,752 LEs available in the Cyclone II EP2C20FPGA which we used.

Figure 18 shows the waveform of the matched filter. As shown, we verified that a correlation signal of the Walsh code was outputted, and the supplied transmission data of an M (maximum length) sequence was observed when a start bit was enabled. In addition, since the maximum processing speed was about $3,000 \mathrm{bps}$, the system could easily realize processing of 2,000 bps, the target transmission speed.

\section{FIELD TRIAL USING AN ACTUAL RAIL}

We conducted a field trial using an actual rail based on the setup shown in Fig. 19. First, we measured the spectral distribution of the CDMA-QAM signal to verify the frequency characteristics of the rail. This result is shown in Fig. 20, which shows that the received signal 
for Iransportation

and Logistics

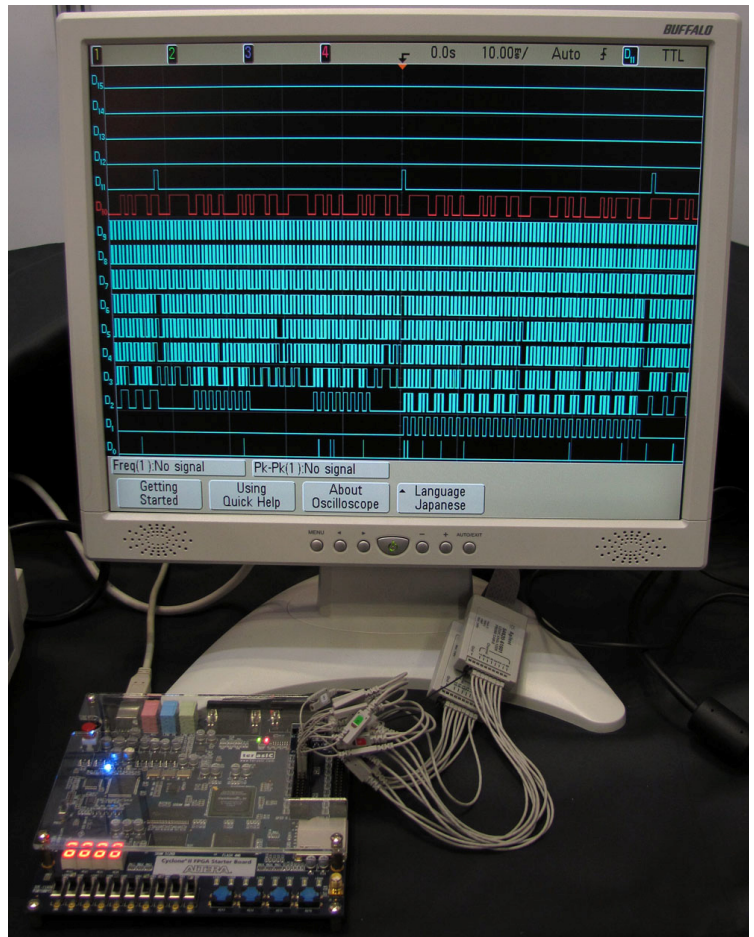

Fig. 17 Developed CDMA receiver using FPGA.

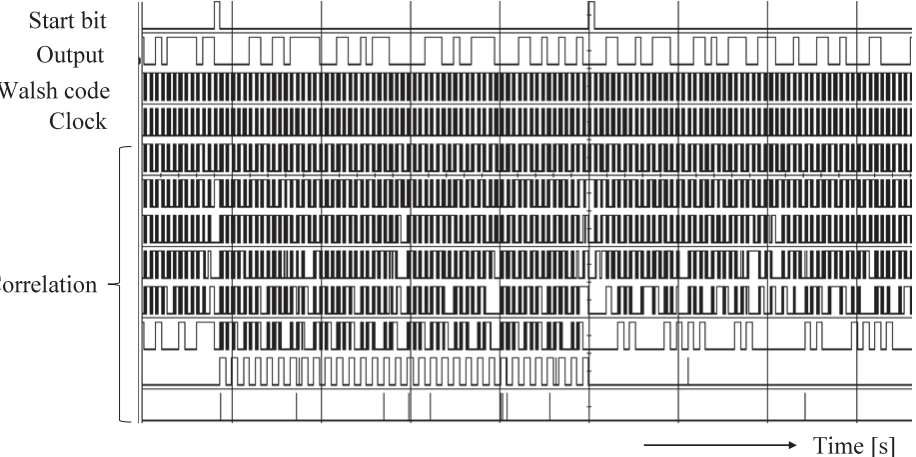

(a) Time scale: $10 \mathrm{~ms}$ div.

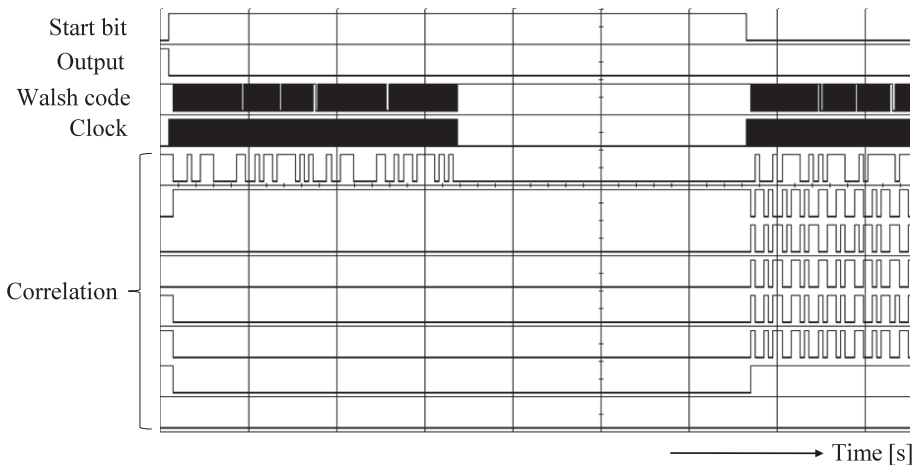

(b) Time scale: $0.1 \mathrm{~ms}$ div.

Fig. 18 Waveform of CDMA receiver. 
and Logistics

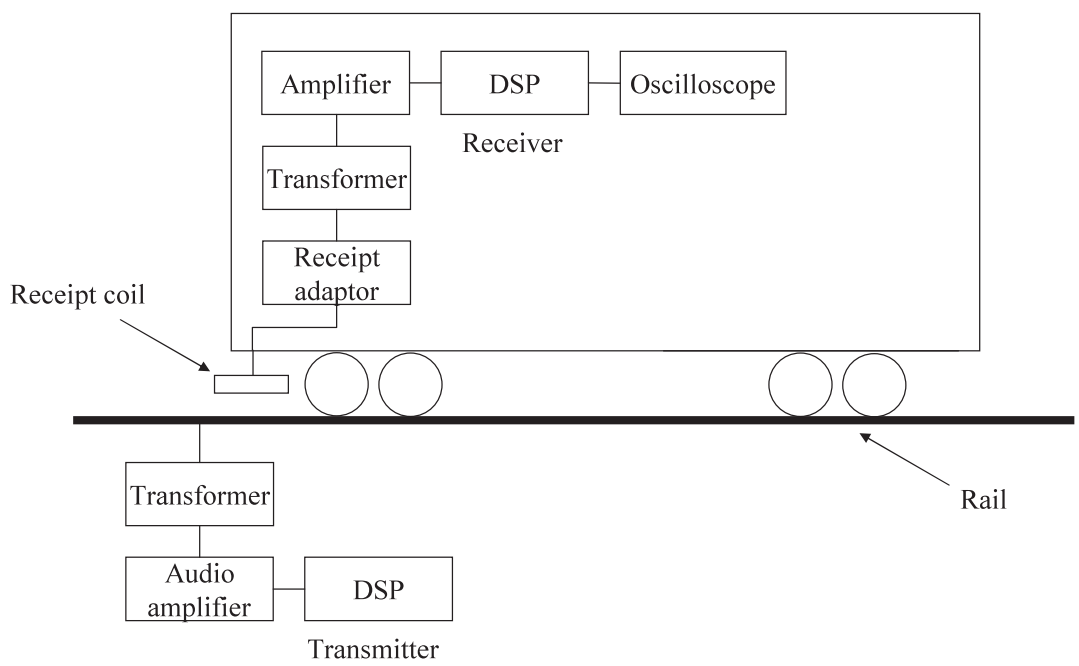

Fig. 19 Setup of field trial using actual rail.

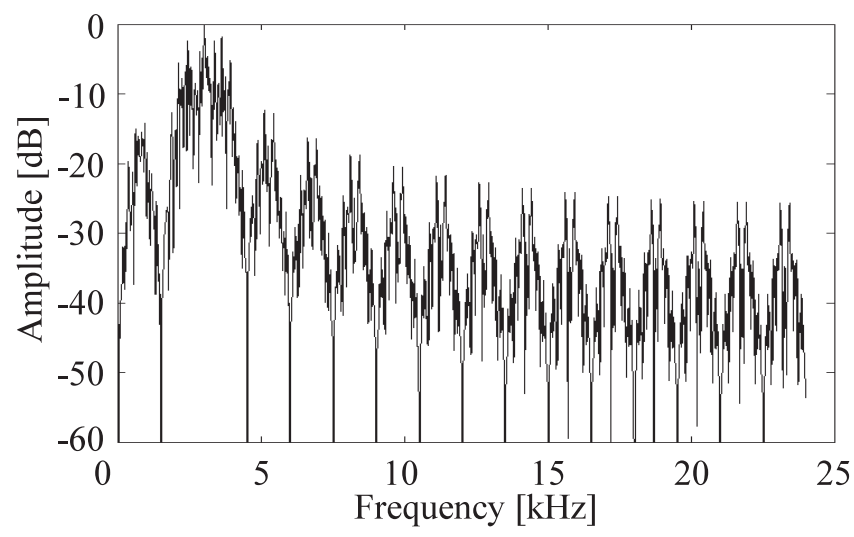

(a) Signal before passing through the rail

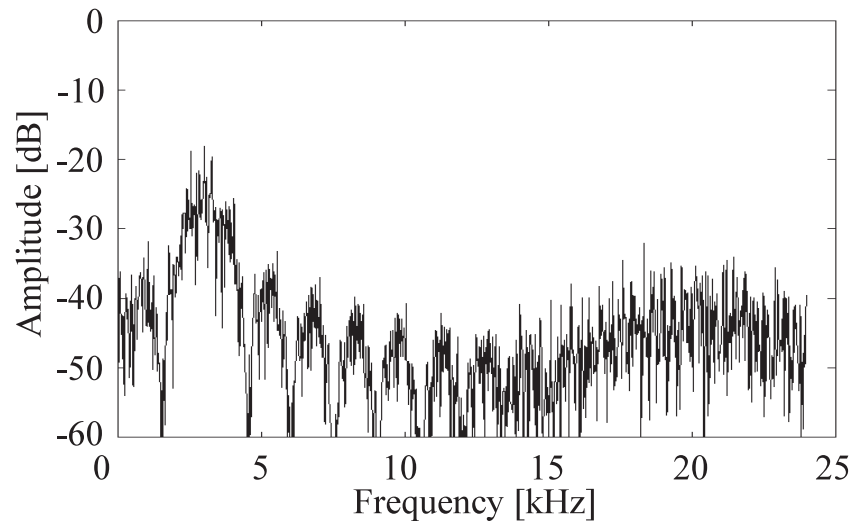

(b) Signal after passing through the rail

Fig. 20 Spectral distribution at the receiver. 


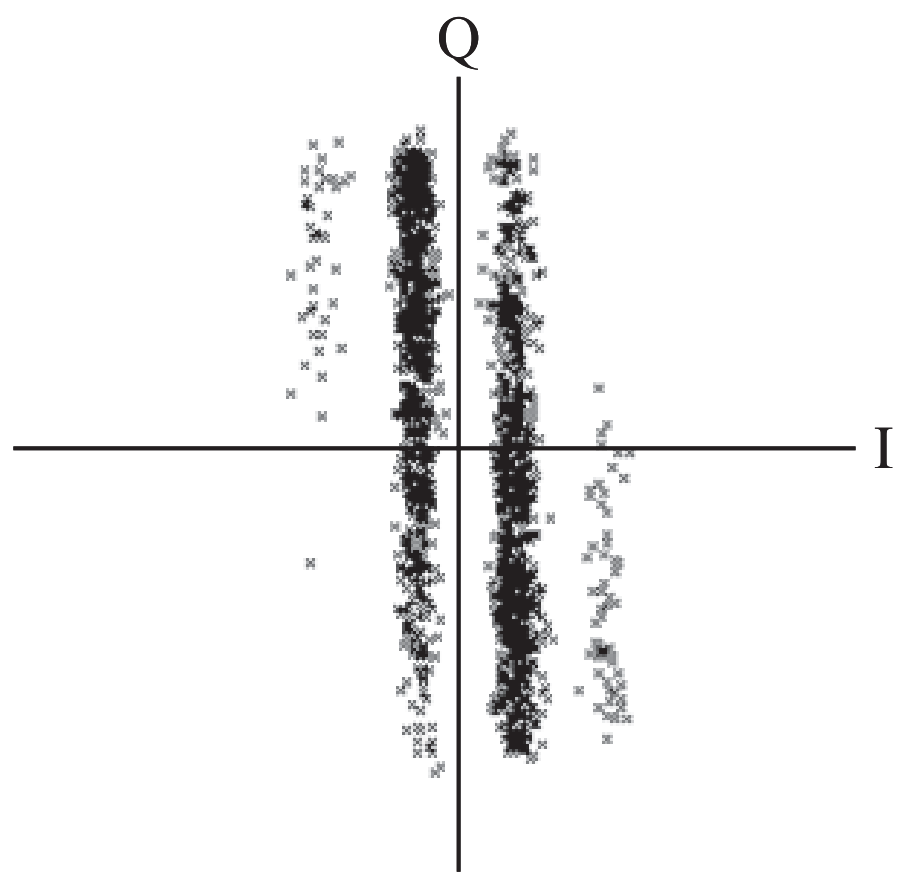

Fig. 21 Constellation characteristics for field trial.

was attenuated by about $20 \mathrm{~dB}$ at the carrier frequency of $3 \mathrm{kHz}$. In view of this characteristic, we employed a software-based automatic gain control (AGC) function in the CDMA-QAM receiver.

Figure 21 shows constellation characteristics when the train was running. From this figure, we observed the constellation characteristics similar to that of $\mathrm{I}: \mathrm{Q}=4: 1$ shown in Fig. 9. As mentioned above, we showed that $\mathrm{I}: \mathrm{Q}=4: 1$ can realize suitable $\mathrm{BER}$ characteristics shown in Fig. 10 by reducing the EVM of the I channel. Therefore, as a result of field trial, we verified that the transceiver achieved suitable constellation characteristics that can achieve suitable BER characteristics after CDMA demodulation processing.

\section{CONCLUSION}

In this paper, we have proposed a novel CDMA-QAM rail transmission system for improving the transmission speed of rail-based signaling.

First, we studied the QAM symbol allocation and designed a symbol allocation scheme in which the symbol interval of the I channel is wider than that of the $Q$ channel. We verified that the BER characteristics improved in proportion to the I/Q symbol interval rate.

Next, we designed the hardware architecture of the CDMA-QAM rail transmission system and developed a CDMA-QAM transceiver using DSPs and an FPGA. We verified that the QAM transceiver successfully performed basic functions in real time, such as synchronous detection. Also, we could dramatically reduce the number of logic elements in the CDMA receiver using time-sharing processing and verified that this receiver outputted a correlation signal of the Walsh code.

In addition, we conducted a field trial using an actual rail. We achieved favorable constellation characteristics for the I channel, which is more important than the Q channel for the BER characteristics.

In Japanese railway, a train control system that a ground device gives the train speed signal directly to each train has been employed for a long time. However, in order to construct more safe and convenient railway systems, it is so important to employ a train control system that trains can realize more flexible control based on the train positioning information given by a ground device. And this control system has been employed in some rail lines at present. 
Our proposed method can give more advanced function to this system by increasing the transmission speed. However, as future work to implement to an actual system, we need to study on a quantitative evaluation such as BER characteristic and safety evaluation of the developed device and so on.

\section{References}

( 1 ) Irie, S. and Hasegawa, T., A study on the Railway Signalling System using Spread Spectrum Communication, Technical Report of the Institute of Electronics, Information and Communication Engineers of Japan, Vol. 93, No. 89 (1993), pp. 43-48.

( 2 ) Mochizuki, H., Asano, A., Sano, M., Takahashi, S. and Nakamura, H., A Study on Railway Signal System to Apply CDMA-QAM Method, Transactions of the Institute of Electrical Engineers of Japan, Vol. 126-D, No. 3 (2006), pp. 345-354.

( 3 ) H. Harada and R. Prasad, Simulation and Software Radio for Mobile Communications, (2002), pp. 90-91, Artech House. 